\title{
A nontargeted study of muscle proteome in severely obese women with androgen excess compared with severely obese men and nonhyperandrogenic women
}

\author{
María Insenser ${ }^{*}$, Rafael Montes-Nieto*, M Ángeles Martínez-García and \\ Héctor F Escobar-Morreale
}

Diabetes, Obesity and Human Reproduction Research Group, Department of Endocrinology and Nutrition, Instituto Ramón y Cajal de Investigación Sanitaria (IRYCIS), Centro de Investigación Biomédica en Red Diabetes y Enfermedades Metabólicas Asociadas (CIBERDEM), Hospital Universitario Ramón y Cajal, Universidad de Alcalá, Carretera de Colmenar km 9,1, E-28034 Madrid, Spain

${ }^{*}$ (M Insenser and R Montes-Nieto contributed equally to this work)

Correspondence should be addressed to H F Escobar-Morreale Email

hectorfrancisco.escobar@ salud.madrid.org

\begin{abstract}
Objective: Androgen excess in women is frequently associated with muscle insulin resistance, especially in obese women with polycystic ovary syndrome. However, whether this is a primary event or the result of indirect mechanisms is currently debated. Design: This is an observational study.

Methods: We obtained skeletal muscle biopsies during bariatric surgery from severely obese men $(n=6)$ and women with $(n=5)$ or without $(n=5)$ androgen excess. We used two-dimensional differential gel electrophoresis and matrix-assisted laser desorption/ionization-time-of-flight/time-of-flight mass spectrometry to identify muscle proteins showing differences in abundance between the groups of obese subjects.

Results: Women with hyperandrogenism presented the lowest abundances of glycogen phosphorylase, pyruvate kinase, $\beta$-enolase, glycerol-3-phosphate dehydrogenase, creatine kinase M-type, and desmin, whereas the abundances of these molecules were similar in control women and men.

Conclusion: According to our nontargeted proteomic approach, women with hyperandrogenism show a specific alteration of the skeletal muscle proteome that could contribute to their insulin resistance. Because men do not show similar results, this alteration does not appear to be the direct effect on muscle of androgen excess, but rather the consequence of indirect mechanisms that merit further studies.
\end{abstract}

\section{Introduction}

The influence of androgens on intermediary metabolism is heavily sex-dependent because both androgen excess in women and androgen deficiency in men facilitate abdominal adiposity, insulin resistance, and related metabolic disorders (1). Moreover, obesity-associated gonadal dysfunction consists of hyperandrogenism in women (2) but hypogonadism in men (3). (c) 2016 European Society of Endocrinology Printed in Great Britain
As the skeletal muscle is the major site of insulinstimulated glucose disposal, this tissue becomes a key to understand the effects of androgens on glucose metabolism and insulin resistance. However, not all effects of androgens on muscle are related to glucose metabolism. The skeletal muscle transcription of several genes related to cytoskeleton, cellular matrix, protein synthesis, or cell

Published by Bioscientifica Ltd 
proliferation is influenced by the balance of sex hormones in healthy men and women (4). Furthermore, skeletal muscle functions as a peripheral endocrine organ by releasing signaling molecules termed myokines, which are implicated in the regulation of several physiological and metabolic pathways $(5,6)$ contributing to energy homeostasis.

Nevertheless, changes in muscle metabolism may be explained not only by a direct effect of androgens but also by indirect effects mediated by other organs such as adipose tissue that under influence by androgens (7), may release signaling molecules that influence muscle metabolism. The interplay between adipose tissue and muscle dysfunction appears to play a central role in the development of metabolic disorders in situations that favor the accumulation of dysfunctional adipose tissue (abdominal adiposity or obesity), and/or decreased muscle mass (age- or sedentarism-associated sarcopenia) (1).

Since the proteome - the set of proteins present in a tissue - is the result of gene expression and epigenetic influences, the study of protein abundance in muscle may provide new insights into the molecular processes that lead to metabolic dysfunction in situations where the concentrations of circulating androgens are altered, an issue that has not been addressed in previous proteomic studies of muscle in insulin resistance-related disorders $(8,9,10)$.
Hence, we aimed to identify differences in the skeletal muscle proteome using biopsies from severely obese control women, women with hyperandrogenism, and men.

\section{Patients and methods}

\section{Subjects}

We obtained skeletal muscle samples from 16 severely obese patients (including five women with hyperandrogenism and five women showing no evidence of hyperandrogenism or reproductive dysfunction, and six reproductively healthy men) during bariatric surgery. Biopsies were obtained from musculus rectus abdominis, which is a typical skeletal muscle with a fiber type distribution similar, for example, to $m$. rectus femoris muscle (11). The subjects selected had similar mean BMI and age. Clinical, hormonal, and biochemical parameters were determined in the fasting state as described earlier $(12,13)$ and are summarized in Table 1 . Fasting glucose and insulin were used for homeostasis model assessment of insulin resistance (HOMA-IR) (14).

All women in the hyperandrogenic group had hyperandrogenemia (serum total testosterone $\geq 2.3 \mathrm{nmol} / 1$ and/or calculated free testosterone $\geq 35 \mathrm{pmol} / \mathrm{l})$. Four of the women in this group had also menstrual dysfunction

Table 1 Clinical, metabolic, and hormonal variables of severely obese subjects from whom skeletal muscle biopsies were obtained during bariatric surgery for proteomic studies. Data are presented as mean \pm s.D. Data were submitted to one-way ANOVA followed by the least significant difference post hoc test.

\begin{tabular}{|c|c|c|c|c|}
\hline & $\begin{array}{c}\text { Control } \\
\text { women }(n=5)\end{array}$ & $\begin{array}{c}\text { Women with } \\
\text { hyperandrogenism }(n=5)\end{array}$ & Men $(n=6)$ & $\boldsymbol{P}$ \\
\hline Age (year) & $36 \pm 7$ & $33 \pm 5$ & $37 \pm 7$ & 0.591 \\
\hline Weight (kg) & $139 \pm 8$ & $137 \pm 15$ & $148 \pm 19$ & 0.468 \\
\hline BMI $\left(\mathrm{kg} / \mathrm{m}^{2}\right)$ & $55 \pm 3$ & $52 \pm 5$ & $50 \pm 6$ & 0.177 \\
\hline Waist-to-hip ratio & $0.78 \pm 0.10$ & $0.86 \pm 0.10$ & $1.04 \pm 0.06^{\dagger \neq}$ & 0.001 \\
\hline Hirsutism score & $1 \pm 1$ & $10 \pm 8^{*}$ & NA & 0.034 \\
\hline Total testosterone $(\mathrm{nmol} / \mathrm{l})$ & $1.9 \pm 0.7$ & $2.7 \pm 1.0$ & $12.8 \pm 2.2^{\dagger \neq}$ & $<0.001$ \\
\hline Free testosterone $(\mathrm{pmol} / \mathrm{l})$ & $35 \pm 10$ & $62 \pm 24 *$ & $340 \pm 55^{\dagger \neq}$ & $<0.001$ \\
\hline SHBG (nmol/l) & $34 \pm 10$ & $22 \pm 14$ & $19 \pm 8$ & 0.089 \\
\hline DHEAS $(\mu \mathrm{mol} / \mathrm{l})$ & $3.0 \pm 1.7$ & $6.3 \pm 1.0$ & $4.4 \pm 2.9$ & 0.095 \\
\hline Androstenedione $(\mathrm{nmol} / \mathrm{l})$ & $8.4 \pm 1.7$ & $12.9 \pm 5.6$ & $9.0 \pm 3.5$ & 0.261 \\
\hline 17-hydroxyprogesterone (nmol/l) & $2.4 \pm 1.2$ & $2.4 \pm 0.9$ & $3.6 \pm 1.8$ & 0.331 \\
\hline Alkaline phosphatase (U/I) & $83 \pm 25$ & $105 \pm 26$ & $73 \pm 21$ & 0.181 \\
\hline Total cholesterol (mmol/l) & $4.5 \pm 1.1$ & $5.5 \pm 0.5$ & $5.6 \pm 1.1$ & 0.130 \\
\hline LDL-cholesterol (mmol/l) & $2.4 \pm 0.6$ & $3.7 \pm 0.6^{*}$ & $3.8 \pm 0.9^{\dagger}$ & 0.023 \\
\hline HDL-cholesterol (mmol/l) & $1.1 \pm 0.3$ & $1.0 \pm 0.1$ & $0.9 \pm 0.2$ & 0.294 \\
\hline Triglycerides (mmol/l) & $1.8 \pm 1.2$ & $2.6 \pm 1.1$ & $1.9 \pm 0.8$ & 0.398 \\
\hline Fasting glucose (mmol/l) & $5.6 \pm 1.9$ & $9.5 \pm 5.9$ & $4.9 \pm 0.8$ & 0.067 \\
\hline Fasting insulin (pmol/l) & $104 \pm 65$ & $146 \pm 91$ & $201 \pm 87$ & 0.197 \\
\hline HOMA-IR & $4.4 \pm 4.4$ & $6.9 \pm 2.8$ & $6.2 \pm 3.0$ & 0.493 \\
\hline
\end{tabular}

HOMA-IR, homeostasis model assessment of insulin resistance; NA, not applicable; SHBG, sex hormone-binding globulin. * $P<0.05$ or less for the difference between women with hyperandrogenism and control women. ${ }^{\dagger} P<0.05$ or less for the difference between men and women with hyperandrogenism. ${ }^{\ddagger} P<0.05$ or less for the difference between men and control women. 
thereby fulfilling the criteria for polycystic ovary syndrome (PCOS) (15) whereas one woman had hirsutism and hyperandrogenemia but showed regular cycles. Ovarian ultrasound was not conducted. Nonhyperandrogenic control women had no clinical or biochemical evidence of androgen excess and had normal menstrual cycles. Men had no symptoms of gonadal or sexual dysfunction. Written informed consent was obtained from all the participants and the study was approved by the local ethics committee.

\section{Sample preparation and protein labeling}

Skeletal muscle samples were obtained during bariatric surgery after a 12 -h overnight fast. Samples were washed immediately in PBS and stored at $-80^{\circ} \mathrm{C}$ until analysis. Proteins were extracted using a Polytron PT-1200C homogenizer with lysis buffer $(8.4 \mathrm{~mol} / \mathrm{l}$ urea, $2.4 \mathrm{~mol} / \mathrm{l}$ thiourea, $50 \mathrm{~g} / \mathrm{l} 3$-((3 cholamidopropyl)dimethylammonio)1-propanesulfonate, $50 \mathrm{mmol} / \mathrm{l}$ dithiothreitol) $(16,17)$. Homogenized tissues were shaken for $1 \mathrm{~h}$ at room temperature and centrifuged at $20000 \mathrm{~g}$ at $4{ }^{\circ} \mathrm{C}$ for $15 \mathrm{~min}$. Interfering elements were removed using 2D Clean-Up Kit (GE Healthcare, Chalfont St Giles, UK), and proteins were resuspended in rehydration buffer $(7 \mathrm{~mol} / \mathrm{l}$ urea, $2 \mathrm{~mol} / \mathrm{l}$ thiourea, $40 \mathrm{~g} / \mathrm{l} 3$-((3 cholamidopropyl) dimethylammonio)-1-propanesulfonate, and $30 \mathrm{mmol} / \mathrm{l}$ Tris(hydroxymethyl)aminomethane hydrochloride $\mathrm{pH}$ 8.5). Protein concentrations were determined using $\mathrm{RD} / \mathrm{DC}$ protein assay (Bio-Rad Laboratories). To evaluate the reproducibility of the extraction, all the samples were separated with $12 \%$ SDS-PAGE, $4 \mu \mathrm{g}$ protein per lane, and silver stained (18).

Solubilized proteins $(50 \mu \mathrm{g})$ were minimally labeled with $400 \mathrm{pmol}$ of either Cy3 or Cy5 fluorescent dye. Protein extracts from individual participants of each group were alternately labeled with Cy3 or Cy5 dyes (to exclude labeling bias). A pool (mixture of equal protein amount of all samples) was labeled with Cy2 dye to serve as an internal standard for normalization in quantitative comparisons. After $30 \mathrm{~min}$ of incubation on ice in the dark, the reaction was stopped by incubation with $1 \mu 1$ $10 \mathrm{mM}$ lysine for $10 \mathrm{~min}$.

\section{Two-dimensional difference in gel electrophoresis, image analysis, and protein identification}

Samples were applied by rehydration in immobilized $\mathrm{pH}$ gradient (IPG) strips ( $24 \mathrm{~cm}, \mathrm{pH} 4-7$, linear) and subjected to isoelectrofocusing in an IPGphor isoelectric focusing
(IEF) system (GE Healthcare, Freiburg, Germany). Strips were transferred to the top of an SDS-PAGE gel and cast on an Ettan DALTsix electrophoresis unit (GE Healthcare). Digital images of gels were acquired using Typhoon 9400 (GE Healthcare), cropped with ImageQuant (GE Healthcare) and analyzed with DeCyder 2D 7.0. (GE Healthcare). Total protein was detected by poststaining of the gels with Coomassie dye (Bio-Rad Laboratories) or silver. Matrix assisted laser desorption ionization-time-of-flight/timeof-flight (MALDI-TOF/TOF) mass spectrometry (MS) analyses for selected spots were performed using a 4800 proteomics analyzer (Applied Biosystems, MDS Sciex, Toronto, Canada).

Detailed information of proteomic techniques, randomization, and combination of samples and MS analysis of protein spots is included in the minimum information about proteomics experiment (MIAPE) document (Supplementary Table 1, see section on supplementary data given at the end of this article).

\section{Experimental design, data analysis and spot selection}

For two-dimensional difference in gel electrophoresis (2DDIGE) experiments, DeCyder's differential in gel analysis (DIA) module was used for intra-gel co-detection of samples and internal standard protein spots, allowing the detection of an average of 750 protein spots on each image with a $7.6 \%$ coefficient of variation. Artifactual spots were filtered and removed.

In the first experiment we quantified the technical variation of the 2D-DIGE technique when using muscle tissue samples. We analyzed pools of all the samples of each group (control women, women with hyperandrogenism, and men) separately in three gels. The first gel served to compare the pooled samples of control women labeled with $\mathrm{Cy} 3$ or $\mathrm{Cy} 5$, the second included the pooled samples of women with hyperandrogenism labeled with Cy3 or Cy5, and the third compared the pool of samples from men labeled with Cy3 or Cy5. A mix of the three pools was labeled with Cy 2 and was used as internal standard to normalize the results. The comparison among the pools labeled with Cy3 and Cy5 using the DIA module gave an estimation of the experimental variability of the 2D-DIGE procedure for each subject subgroup. The thresholds for the fold-differences in protein abundance warranting with 95\% confidence that a difference between the groups being compared did not result from experimental variation were 1.4-fold for control women, 1.5-fold for women with hyperandrogenism and 1.3-fold for men. Therefore, we selected a 1.4-fold difference as the cut-off that 
excluded experimental variability when comparing muscle in further experiments.

In the second experiment we evaluated the differences in protein abundance in muscle biopsies from control women, women with hyperandrogenism and men. Half of the samples of each group were labeled with $\mathrm{Cy} 3$ and the other half were labeled with Cy5 to avoid biases in labeling efficiency. A pool of all the samples labeled with Cy2 served as internal standard for normalization.

The 24 images from the eight gels were submitted to the biological variation analysis (BVA) module. Based on spot volumes, BVA performed an inter-gel matching that allowed a further comparative cross-gel statistical analysis to detect spots showing different abundance in the groups. The differences between the abundances from two samples are reported as ratios. An average of 310 spots was matched, and their abundances were normalized and quantified. Only spots present in at least $80 \%$ of the gel images were submitted for further analyses. Finally, spot matching and data quality were verified manually to exclude artifacts and to avoid false positives.

We decided against applying a priori statistical correction techniques such as q-values to estimate the rate of false discovery at a given value of $P$, because this approach, aiming to decrease the probability of false discoveries (type 1 errors), would at the same time increase the probability of missing true results (type 2 errors). The latter is a particularly important concern for our study because the number of proteins presenting with differences in abundance with our proteomic untargeted discovery approach is usually small. Instead, we focused on reporting only differences that we were sure did not result from the technical variability of our technique. Hence, we submitted for MS identification only spots showing differences in protein abundance that were not only statistically significant at $\alpha=0.05$ level but were also $\geq 1$.4-fold in magnitude, thereby excluding any technical variation as their possible cause.

\section{Gene expression analysis}

Muscle samples were washed in PBS and immediately stored at $-80^{\circ} \mathrm{C}$ until submitted for ribonucleic acid (RNA) extraction. Total RNA was isolated from $25 \mathrm{mg}$ of muscle tissue with an RNeasy Fibrous Tissue Mini Kit according to manufacturer's guidelines (Qiagen). The concentration and quality of the extracted RNA were assessed by measuring the 260/280 and 260/230 absorbance ratios in a NanoDrop 2000 spectrophotometer (Thermo Scientific, Wilmington, DE, USA).
First-strand cDNA was synthesized using equal amounts of total RNA with SuperScript Vilo MasterMix (Invitrogen, Life Technologies) following manufacturer's instructions. Due to the limited quantity of samples a TaqMan PreAmp Master Mix Kit (Applied Biosystems) was used to increase the amount of specific cDNA targets as described (19). Prevalidated TaqMan Gene Expression assays were used for the relative quantification of $P K M$ (Assay ID: Hs00761782_s1), PYGM (Hs00989942_m1), ENO3 (Hs01093275_m1), GPD1 (Hs01100039_m1), CKM (Hs00176490_m1), and DES (Hs00157258_m1), in a StepOne Plus Real-Time PCR instrument using the StepOne Software v2.1 (Applied Biosystems). Cyclophilin A (PPIA; 4333763F) was used as an endogenous control to normalize target gene expression in each sample (20). A threshold cycle $(\mathrm{Ct})$ was obtained for each amplification curve, and a $\Delta C t$ value was first calculated by subtracting the $C t$ value for cyclophilin A from the $C t$ value of the specific transcript. Data were expressed as arbitrary units of expression $\left(\log _{2}^{-\Delta C t}\right)$. All samples were performed in triplicate, and negative controls were included in all the reactions.

\section{Western blot analyses}

1D and 2D SDS-PAGE western blot analysis was performed using standard techniques and equipment (Bio-Rad Laboratories). In 1D SDS-PAGE western blot analysis $20 \mu \mathrm{g}$ of skeletal muscle proteins were loaded in $10 \%$ polyacrylamide gel. In 2D SDS-PAGE western blot analysis $100 \mu \mathrm{g}$ of skeletal muscle proteins were separated on $7-\mathrm{cm}$ linear IPG strips $\mathrm{pH} 4-7$ by IEF prior to seal on top of $10 \%$ polyacrylamide gel with $0.5 \%$ agarose. Proteins from both $1 \mathrm{D}$ and $2 \mathrm{D}$ gels were separated at $150 \mathrm{~V}$ for $50 \mathrm{~min}$ until the front of the bromophenol blue dye reached the bottom of the gels and were transferred to nitrocellulose membranes $(100 \mathrm{~mA}, 1 \mathrm{~h})$. Membranes were blocked in Tris-buffered saline containing 5\% dry non-fat milk for $1 \mathrm{~h}$. Immunoblotting was performed with 1:4000 rabbit polyclonal pyruvate kinase antibody (anti-PKM2 ab137791; Abcam, Cambridge, UK), 1:170 mouse monoclonal glycogen phosphorylase antibody (anti-PYGM ab88078), 1:2000 mouse monoclonal desmin antibody (anti-desmin ab6322), 1:2000 rabbit polyclonal glycerol3-phosphate dehydrogenase antibody (anti-GPD1 ab153902), 1:4000 mouse monoclonal creatine kinase antibody (anti-CKM ab54637), and 1:12 000 rabbit polyclonal $\beta$-enolase antibody (anti-ENO3 ab126259). 1:4000 rabbit polyclonal cytochrome c oxidase subunit four antibody (anti-COXIV ab16056) was utilized as the 
loading control in 1D gels and 1:1000 rabbit polyclonal actin (anti-actin ab52218) in 2D gels. Detection was performed with secondary antibodies conjugated to IRDye $800 \mathrm{CW}$ or IRDye $680 \mathrm{RD}$ and densitometric analysis used the Odyssey Infrared Imaging System V.3.0 (LI-COR; Biosciences, Lincoln, NE, USA).

\section{Statistical analysis}

Data are expressed as mean \pm s.D. unless otherwise stated. After testing the normal distribution of the continuous variables by the Kolmogorov-Smirnov test, we applied logarithmic transformation as needed to ensure normality of skewed variables. Data from control women, women with hyperandrogenism and men were compared by oneway ANOVA followed by post hoc tests as described. Discontinuous variables were compared by $\chi^{2}$ test.

The relationships among the proteins identified here, free testosterone and HOMA-IR were analyzed using Spearman's correlation. We used PASW Statistics 18 (SPSS, Chicago, IL, USA) for analyses. $P<0.05$ was considered statistically significant.

\section{Results}

\section{Clinical, biochemical, and hormonal variables of the subjects included in the study}

The variables included in the study are summarized in Table 1. As expected from the design, no differences were found in the age and BMI among the groups. Rates of metabolic complications (hypertension, dyslipidemia, or diabetes) were similar in these groups (3/5 in control women, 3/5 in women with hyperandrogenism, and 3/6 in men; $\left.\chi^{2}=0.152, P=0.927\right)$. Women with hyperandrogenism showed higher hirsutism scores compared with control women. Total and free testosterone concentrations and waist-to-hip ratio were higher, whereas sex hormone-binding globulin (SHBG) concentration was lower, in men compared with control women. Hyperandrogenic women showed values that were in-between those of control women and men, including higher free testosterone concentrations when compared with control women. Fasting glucose and insulin concentrations and HOMA-IR were not statistically different between the groups, however control women showed the lowest surrogate indexes of insulin resistance of all groups, but did not reach statistical significance possibly because of the small sample size of the groups compared here. No differences were observed in lipid profiles with the exception of LDL-cholesterol concentrations which was lower in control women compared to men and women with hyperandrogenism.

\section{Proteome analysis of skeletal muscle samples}

We found differences in the protein abundance of seven spots. The logarithms of the standardized protein abundance generated by DeCyder were submitted to one-way ANOVA followed by Bonferroni correction in order to identify the individual groups causing the differences.

Excision, in-gel digestion and MALDI-TOF/TOF analysis of these spots allowed us the identification of the proteins present in six of the seven spots (four were identified matching their peptide mass fingerprint in Mascot database and two were identified using tandem mass spectrometry). The seventh spot could not be identified, probably because of the low amount of protein present in the gels (Fig. 1). Detailed information of the identification process of the six spots is summarized in Table 2, including accession numbers, theoretical molecular weight, isoelectric point values, DeCyder software, and parameters of the identification method, and results of statistical analysis.

Women with hyperandrogenism showed the lowest abundance of the six proteins identified (Table 2 and Fig. 2).

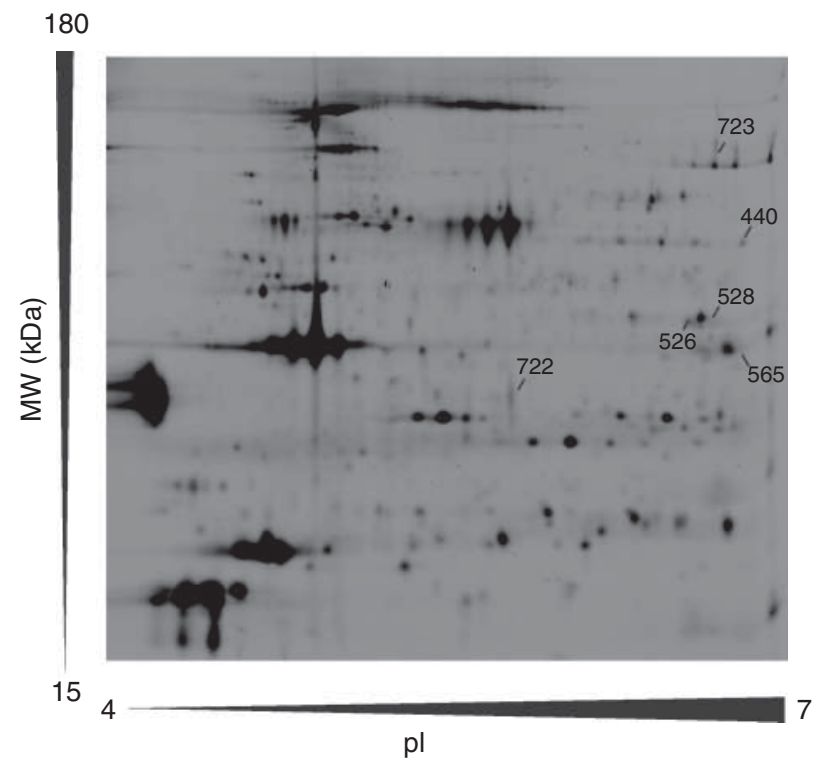

\section{Figure 1}

Representative 2D-DIGE map of skeletal muscle protein extracts including the pick locations of the proteins identified in this study. 
The abundance of pyruvate kinase was lower in hyperandrogenic women compared with control women and men, whereas their abundance of glycogen phosphorylase was lower compared with control women, with men showing intermediate values that were not different compared with both groups of women. The abundances of $\beta$-enolase, glycerol-3-phosphate dehydrogenase, creatine kinase M-type, and desmin were lower in hyperandrogenic women compared with men, with control women presenting with intermediate abundances that were not different compared with the other groups. We also analyzed possible sex-dependent differences in protein abundance, considering as a whole obese women with or without androgen excess. $\beta$-enolase $(P=0.01)$, glycerol-3-phosphate dehydrogenase $(P=0.03)$ and desmin $(P=0.02)$ were more abundant in men compared with women, whereas no differences were observed in pyruvate kinase, glycogen phosphorylase, and creatine kinase M-type. With the exception of desmin, which is a structural protein, all the proteins identified in our study have catalytic activity and are involved in muscle energy metabolism.

\section{Validation of the differences found in protein abundance}

To verify the 2D-DIGE results, we submitted the 16 skeletal muscle samples to gene expression analysis by real-time quantitative PCR and to $1 \mathrm{D}$ and $2 \mathrm{D}$ SDS-PAGE western blot analyses. We analyzed pyruvate kinase, glycogen phosphorylase, $\beta$-enolase, glycerol-3phosphate dehydrogenase, creatine kinase M-type, and desmin. With the exception of desmin, the lowest gene expression and protein abundance for the molecules identified here were those of hyperandrogenic women, yet the relatively large dispersion of the data precluded that the apparent differences with respect to the values found in control women and men reached statistical significance (Fig. 3).

To avoid this limitation, we aimed to confirm the differences in creatine kinase M-type and $\beta$-enolase protein abundance by a 2D SDS-PAGE western blot analysis in which pools of the samples of all individuals in each group were compared (Fig. 4). The abundances of creatine kinase M-type (spot 565 in 2D-DIGE) and of two protein species of $\beta$-enolase (spots 519 and 528 in 2DDIGE) appeared to be reduced in hyperandrogenic women compared with control women, and the abundances in two protein species of $\beta$-enolase appeared to be also lower in hyperandrogenic women compared with men (Fig. 4). 

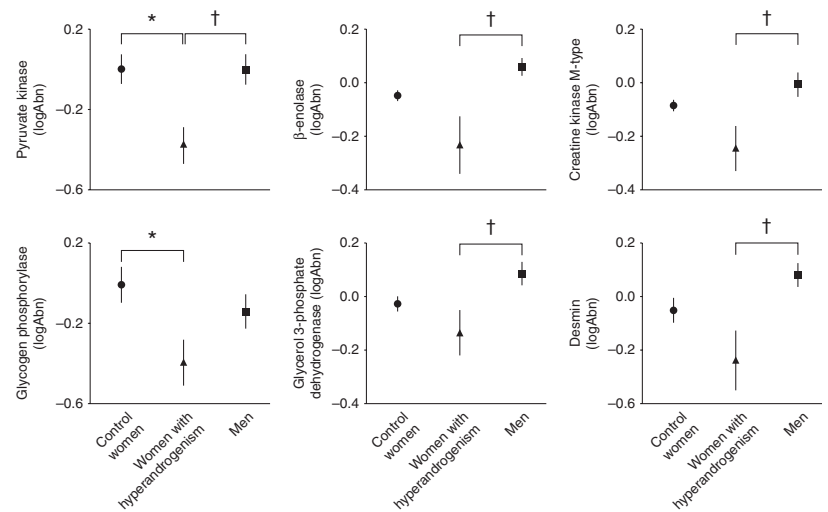

\section{Figure 2}

Differences between control women, women with hyperandrogenism, and men in the protein abundance of skeletal muscle (logarithm of abundance (logAbn)) as studied by 2D-DIGE. Data are means \pm s.E.M. ${ }^{*} P<0.05$ or less for differences between women with hyperandrogenism and control women. ${ }^{\dagger} P<0.05$ or less for the difference between men and women with hyperandrogenism.

\section{Correlations of protein abundance with clinical variables}

In order to identify the proteins that might be related with androgen concentrations and insulin resistance we analyzed the correlations of the protein abundances with free testosterone and HOMA-IR. The abundances of pyruvate kinase and glycogen phosphorylase correlated negatively with HOMA-IR $(\rho=-0.631, P=0.016$, and $\rho=-0.535, P=0.033$ respectively), but we did not find correlations between the abundances of any of the proteins identified in the study and free testosterone (data not shown).

\section{Discussion}

Our present nontargeted proteomic analysis of the protein content of skeletal muscle indicates that severely obese women with androgen excess present with reduced abundances of several enzymes related to muscle, glycolysis, glycogenolysis, and energy expenditure, suggesting a certain dysregulation of energy metabolism. This abnormality appears to be specific of hyperandrogenic women given that it was not present in similarly obese control women and men, and might be related to the skeletal muscle insulin resistance characteristic of PCOS (21). Since insulin facilitates muscle glycolysis, the decrease in the abundance of glycolytic enzimes is in conceptual agreement with reduced insulin signaling in the muscle of hyperandrogenic obese women, Glycogen phosphorylase catalyzes the rate-limiting step of glycogenolysis; pyruvate kinase catalyzes the final, and $\beta$-enolase the penultimate, steps of glycolysis; glycerol-3phosphate dehydrogenase is responsible for maintaining the redox potential across the inner mitochondrial membrane in glycolysis and also serves as a link between carbohydrate and lipid metabolism by generating glycerol-3-phosphate; and creatine kinase M-type plays a central role in the muscle energy transduction needed for glycolysis. Moreover, the decreased abundance of desmin - a muscle-specific intermediate filament involved in cytoskeleton structure that may act as a mechanosignal transducer allowing muscle fibers to sense contractile
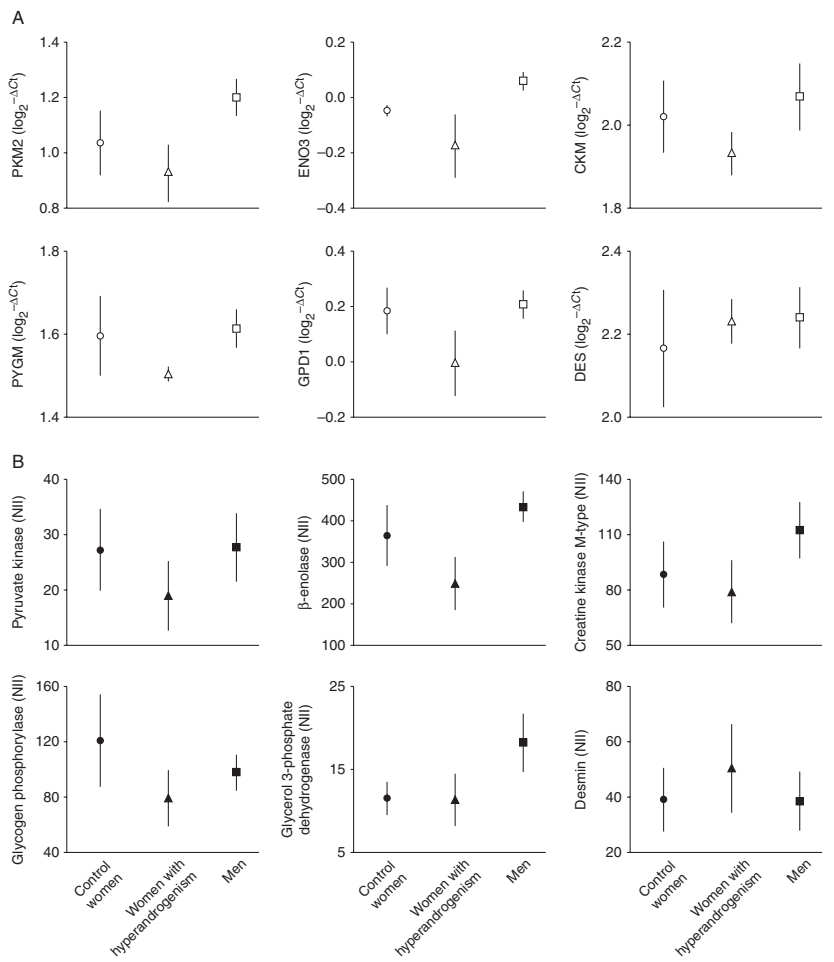

\section{Figure 3}

Validation of 2D-DIGE experiments. (A) mRNA expression analysis $\left(\log _{2}{ }^{-\Delta C t}\right)$ of the genes encoding the skeletal muscle proteins identified by 2D-DIGE. Data are means \pm s.E.M. CKM, creatine kinase M-type; DES, desmin; ENO3, $\beta$-enolase; GPD1, glycerol 3-phosphate dehydrogenase; $P K M 2$, pyruvate kinase; PYGM, glycogen phosphorylase. (B) Abundance of the proteins (normalized integrated intensity (NII)) identified by 2D-DIGE in skeletal muscle samples as studied by 1D western-blot. Data are means \pm s.E.M. 

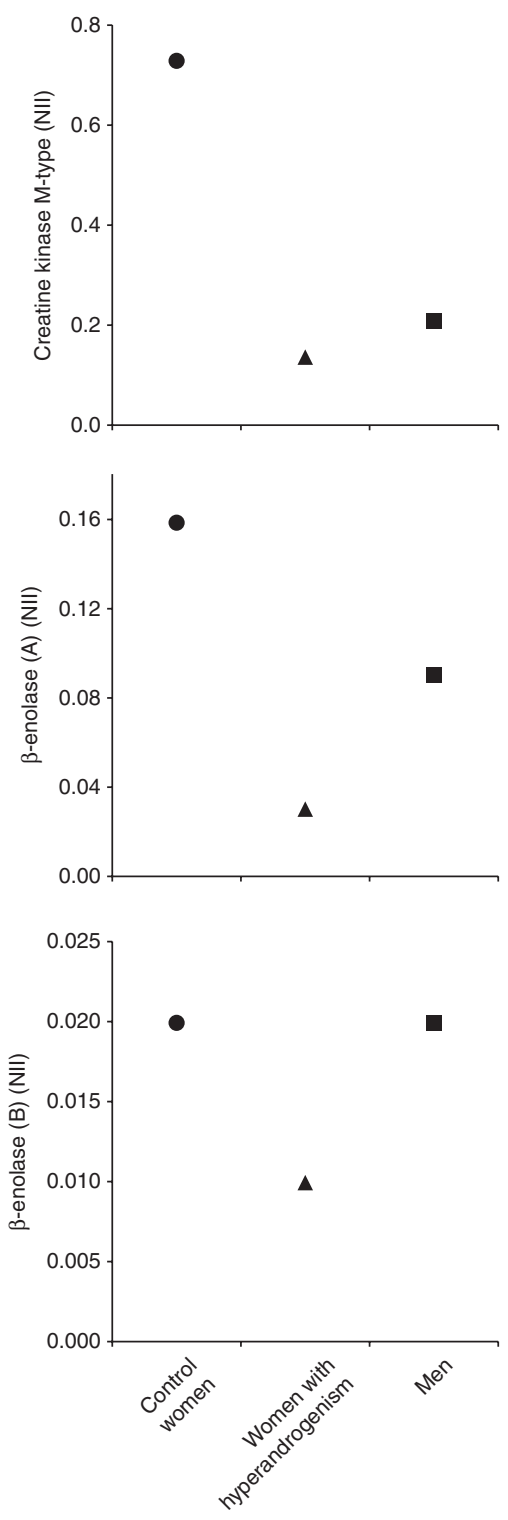

\section{Figure 4}

Validation of 2D-DIGE experiments. The abundances of creatine kinase and two isoforms of $\beta$-enolase were assessed in pools of samples of the five control women, of the five women with hyperandrogenism, and of the six men using $2 \mathrm{D}$ western-blot analysis. NII, normalized integrated intensity.

activity and respond appropriately - has been also found in association with obesity and type 2 diabetes, influencing insulin resistance $(22,23)$.

However, the dysregulation in muscle energy enzymes of women with hyperandrogenism does not appear to be the direct result of their androgen excess; otherwise we would have found a similar decrease in the abundance of these enzymes in men, but their protein abundances were similar of those of control women and considerably higher than those observed in hyperandrogenic women. In accordance, the abundances of pyruvate kinase and glycogen phosphorylase showed strong negative correlations with HOMA-IR, but not with free testosterone concentrations.

Even though animal models suggested that exposure of cultured rat myotubes to testosterone might promote insulin resistance by facilitating the insulin-stimulated phosphorylation of several molecules involved in insulin signaling (24), human studies using cultured myotubes from patients with PCOS and healthy women have demonstrated that the mechanisms governing insulin resistance in skeletal muscle of PCOS patients in vivo are not primarily defective (25) because even the direct administration of testosterone to these myotubes does not influence insulin sensitivity (26). Together with our present proteomic results, these findings suggest that if androgen excess is implicated in the muscular insulin resistance in PCOS, their effects are probably mediated by indirect mechanisms (26).

Our present cross-sectional experimental design does not permit identifying these indirect mechanisms, but we suspect that obesity and abdominal adiposity might be a link between female androgen excess and muscle insulin resistance based on previous studies from our laboratory $(27,28)$. Obesity is definitely involved in the development of muscle insulin resistance in androgen excess disorders. A recent non-targeted gas chromatography-mass spectrometry metabolomic study indicated that even though central (hepatic) insulin resistance was present in both lean and obese patients with PCOS, the metabolic profiles of lean patients suggest conserved insulin sensitivity in muscle and adipose tissue whereas the opposite occurred in obese patients, in whom whole-body insulin resistance was apparent (27).

Patients with PCOS are characterized by global and abdominal adiposity $(28,29)$ when compared with healthy women and men: women with PCOS have higher global adiposity and increased amounts of visceral adipose tissue compared with control women, and even though men have higher total amounts of body fat than women, the amounts of body fat relative to total body mass are higher in patients with PCOS than in men because of the much larger lean body mass of the latter (28). Recent targeted and non-targeted studies suggest that not only the distribution of body fat, but also the function of visceral adipose tissue, is altered in women with PCOS to the extent of resembling that of men and not that of healthy women $(7,19)$. 
In our present small series of severely obese patients the waist-to-hip ratio of women with hyperandrogenism was higher compared to control women, indicating abdominal adiposity. We might speculate if the abdominal adiposity characteristic of hyperandrogenic women, possibly through the dysfunctional secretion of adipokines and other mediators, could influence muscle metabolism reducing the expression and protein abundance of enzymes involved in energy metabolism, leading to the abovementioned muscle insulin resistance. On the contrary, obese healthy men could be protected against the deleterious effects of abdominal adiposity on muscle function because of their much larger muscle mass (1). To this regards, the abovementioned studies in the rat suggested interaction between testosterone and insulin on muscle insulin signaling consisting in low-dose testosterone - leading to mildly increased androgen levels such as might happen in women with functional hyperandrogenism - resulted into altered insulin signaling in female cultured myotubes, but higher testosterone doses - leading to androgen levels similar to that of male animals - ameliorated such negative effects (4). This finding may suggest the possibility of sexual dimorphism in the effects of androgens on muscle insulin signaling, and might contribute to explain why men maintain muscle insulin sensitivity despite very high testosterone concentrations and develop insulin resistance when such concentrations are decreased. The study of the proteomic profile of muscle samples from obese men with androgen deficiency might have shed light into this controversial issue yet, unfortunately, we had no access to these samples for the present study.

Even though the use of 2D-DIGE techniques in our study allowed an accurate quantification of changes in protein abundance that might result from posttranslational modifications, alternative transcription start sites, or alternative splice variants, this technique is not free of limitations such as problematic identification of proteins showing very low abundance, extreme isoelectric point and/or low molecular weight, or very hydrophobic proteins. Moreover, our study may have been underpowered to detect differences in the abundance of other proteins. Hence, with our experimental design we cannot exclude a wider alteration of muscle proteome in obese women with hyperandrogenism.

In conclusion, obese hyperandrogenic women present with reduced abundance of enzymes and structural proteins involved in energy metabolism that might contribute to skeletal insulin resistance. However, these findings do not appear to be the direct consequence of androgen excess because men do not show any of these changes in protein abundance. Our present results may serve as the basis for future studies addressing the indirect mechanisms that induce specific muscle proteome abnormalities in women with hyperandrogenism.

\section{Supplementary data}

This is linked to the online version of the paper at http://dx.doi.org/10.1530/ EJE-15-0912.

Declaration of interest

The authors declare that there is no conflict of interest that could be perceived as prejudicing the impartiality of the research reported.

\section{Funding}

Supported by grants PI1100357 and PI1501686 from Instituto de Salud Carlos III, Spanish Ministry of Economy and Competitiveness. Centro de Investigación Biomédica en Red Diabetes y Enfermedades Metabólicas Asociadas is also an initiative of Instituto de Salud Carlos III. Supported in part by Fondo Europeo de Desarrollo Regional FEDER (European Union).

\section{Author contribution statement}

M Insenser, R Montes-Nieto, and M Á Martínez-García. researched data, drafted the manuscript, and contributed critically to discussion. H F Escobar-Morreale designed the study, researched data, analyzed the results and wrote the final version of the manuscript. All authors approved the decision to submit the manuscript and take public responsibility for its whole content.

\section{References}

1 Escobar-Morreale HF, Alvarez-Blasco F, Botella-Carretero JI \& Luque-Ramirez M. The striking similarities in the metabolic associations of female androgen excess and male androgen deficiency. Human Reproduction 201429 2083-2091. (doi:10.1093/humrep/ deu198)

2 Escobar-Morreale HF, Botella-Carretero JI, Alvarez-Blasco F, Sancho J \& San Millan JL. The polycystic ovary syndrome associated with morbid obesity may resolve after weight loss induced by bariatric surgery. Journal of Clinical Endocrinology and Metabolism 200590 6364-6369. (doi:10.1210/jc.2005-1490)

3 Calderon B, Galdon A, Calanas A, Peromingo R, Galindo J, Garcia-Moreno F, Rodriguez-Velasco G, Martin-Hidalgo A, Vazquez C, Escobar-Morreale HF et al. Effects of bariatric surgery on male obesity-associated secondary hypogonadism: comparison of laparoscopic gastric bypass with restrictive procedures. Obesity Surgery 201424 1686-1692. (doi:10.1007/s11695-014-1233-y)

4 Yoshioka M, Boivin A, Bolduc C \& St-Amand J. Gender difference of androgen actions on skeletal muscle transcriptome. Journal of Molecular Endocrinology 200739 119-133. (doi:10.1677/JME-07-0027)

5 Pedersen BK \& Febbraio MA. Muscles, exercise and obesity: skeletal muscle as a secretory organ. Nature Reviews. Endocrinology 20128 457-465. (doi:10.1038/nrendo.2012.49)

6 Roca-Rivada A, Al-Massadi O, Castelao C, Senín LL, Alonso J, Seoane LM, García-Caballero T, Casanueva FF \& Pardo M. 
Muscle tissue as an endocrine organ: Comparative secretome profiling of slow-oxidative and fast-glycolytic rat muscle explants and its variation with exercise. Journal of Proteomics 201275 5414-5425. (doi:10.1016/j.jprot.2012.06.037)

7 Montes-Nieto R, Insenser M, Martinez-Garcia MA \& EscobarMorreale HF. A nontargeted proteomic study of the influence of androgen excess on human visceral and subcutaneous adipose tissue proteomes. Journal of Clinical Endocrinology and Metabolism 201398 E576-E585. (doi:10.1210/jc.2012-3438)

8 Gelfi C, Vasso M \& Cerretelli P. Diversity of human skeletal muscle in health and disease: contribution of proteomics. Journal of Proteomics 201174 774-795. (doi:10.1016/j.jprot.2011.02.028)

9 Hittel DS, Hathout Y, Hoffman EP \& Houmard JA. Proteome analysis of skeletal muscle from obese and morbidly obese women. Diabetes 2005 54 1283-1288. (doi:10.2337/diabetes.54.5.1283)

10 Kim DH, Choi JW, Joo JI, Wang X, Choi DK, Oh TS \& Yun JW. Changes in expression of skeletal muscle proteins between obesity-prone and obesity-resistant rats induced by a high-fat diet. Journal of Proteome Research 201010 1281-1292. (doi:10.1021/ pr101048q)

11 Johnson MA, Sideri G, Weightman D \& Appleton D. A comparison of fibre size, fibre type constitution and spatial fibre type distribution in normal human muscle and in muscle from cases of spinal muscular atrophy and from other neuromuscular disorders. Journal of the Neurological Sciences 197320 345-361. (doi:10.1016/0022-510X (73)90169-X)

12 Escobar-Morreale HF, Lasuncion MA \& Sancho J. Treatment of hirsutism with ethinyl estradiol-desogestrel contraceptive pills has beneficial effects on the lipid profile and improves insulin sensitivity. Fertility and Sterility $2000 \mathbf{7 4}$ 816-819. (doi:10.1016/S00150282(00)00718-4)

13 Escobar-Morreale HF, Sanchon R \& San Millan JL. A prospective study of the prevalence of nonclassical congenital adrenal hyperplasia among women presenting with hyperandrogenic symptoms and signs. Journal of Clinical Endocrinology and Metabolism 200893 527-533. (doi:10.1210/jc.2007-2053)

14 Matthews DR, Hosker JP, Rudenski AS, Naylor BA, Treacher DF \& Turner RC. Homeostasis model assessment: insulin resistance and $\beta$-cell function from fasting plasma insulin and glucose concentrations in man. Diabetologia 198528 412-419. (doi:10.1007/BF00280883)

15 Azziz R, Carmina E, Dewailly D, Diamanti-Kandarakis E, EscobarMorreale HF, Futterweit W, Janssen OE, Legro RS, Norman RJ, Taylor A et al. Positions statement: criteria for defining polycystic ovary syndrome as a predominantly hyperandrogenic syndrome: an Androgen Excess Society guideline. Journal of Clinical Endocrinology and Metabolism 200691 4237-4245. (doi:10.1210/jc.2006-0178)

16 Corton M, Villuendas G, Botella JI, San Millan JL, Escobar-Morreale HF $\&$ Peral B. Improved resolution of the human adipose tissue proteome at alkaline and wide range $\mathrm{pH}$ by the addition of hydroxyethyl disulfide. Proteomics 20044 438-441. (doi:10.1002/pmic.200300644)

17 Corton M, Botella-Carretero JI, Lopez JA, Camafeita E, San Millan JL, Escobar-Morreale HF \& Peral B. Proteomic analysis of human omental adipose tissue in the polycystic ovary syndrome using two-dimensional difference gel electrophoresis and mass spectrometry. Human Reproduction 200823 651-661. (doi:10.1093/humrep/dem380)
18 Blum H, Beier H \& Gross HJ. Improved silver staining of plant proteins, RNA and DNA in polyacrylamide gels. Electrophoresis 1987 8 93-99. (doi:10.1002/elps.1150080203)

19 Martinez-Garcia MA, Montes-Nieto R, Fernandez-Duran E, Insenser M, Luque-Ramirez M \& Escobar-Morreale HF. Evidence for masculinization of adipokine gene expression in visceral and subcutaneous adipose tissue of obese women with polycystic ovary syndrome (PCOS). Journal of Clinical Endocrinology and Metabolism 201398 E388-E396. (doi:10.1210/jc.2012-3414)

20 Neville MJ, Collins JM, Gloyn AL, McCarthy MI \& Karpe F. Comprehensive human adipose tissue mRNA and microRNA endogenous control selection for quantitative real-time-PCR normalization. Obesity 201119 888-892. (doi:10.1038/oby.2010.257)

21 Dunaif A, Wu X, Lee A \& Diamanti-Kandarakis E. Defects in insulin receptor signaling in vivo in the polycystic ovary syndrome (PCOS). American Journal of Physiology. Endocrinology and Metabolism 2001281 E392-E399.

22 Hwang H, Bowen BP, Lefort N, Flynn CR, De Filippis EA, Roberts C, Smoke CC, Meyer C, Hojlund K, Yi Z et al. Proteomics analysis of human skeletal muscle reveals novel abnormalities in obesity and type 2 diabetes. Diabetes 201059 33-42. (doi:10.2337/db09-0214)

23 Coletta DK \& Mandarino LJ. Mitochondrial dysfunction and insulin resistance from the outside in: extracellular matrix, the cytoskeleton, and mitochondria. American Journal of Physiology. Endocrinology and Metabolism 2011301 E749-E755. (doi:10.1152/ ajpendo.00363.2011)

24 Allemand MC, Irving BA, Asmann YW, Klaus KA, Tatpati L, Coddington CC \& Nair KS. Effect of testosterone on insulin stimulated IRS1 Ser phosphorylation in primary rat myotubes - a potential model for PCOS-related insulin resistance. PLoS ONE 20094 e4274. (doi:10.1371/journal.pone.0004274)

25 Eriksen M, Porneki AD, Skov V, Burns JS, Beck-Nielsen H, Glintborg D \& Gaster M. Insulin resistance is not conserved in myotubes established from women with PCOS. PLoS ONE 20115 e14469. (doi:10.1371/ journal.pone.0014469)

26 Eriksen MB, Glintborg D, Nielsen MF, Jakobsen MA, Brusgaard K, Tan Q $\&$ Gaster M. Testosterone treatment increases androgen receptor and aromatase gene expression in myotubes from patients with PCOS and controls, but does not induce insulin resistance. Biochemical and Biophysical Research Communications 2014451 622-626. (doi:10.1016/j.bbrc.2014.08.033)

27 Escobar-Morreale HF, Samino S, Insenser M, Vinaixa M, LuqueRamirez M, Lasuncion MA \& Correig X. Metabolic heterogeneity in polycystic ovary syndrome is determined by obesity: plasma metabolomic approach using GC-MS. Clinical Chemistry 201258 999-1009. (doi:10.1373/clinchem.2011.176396)

28 Borruel S, Fernandez-Duran E, Alpañes M, Martí D, Alvarez-Blasco F, Luque-Ramirez M \& Escobar-Morreale HF. Global adiposity and thickness of intraperitoneal and mesenteric adipose tissue depots are increased in women with polycystic ovary syndrome (PCOS). Journal of Clinical Endocrinology and Metabolism 201398 1254-1263. (doi:10.1210/ jc.2012-3698)

29 Barber TM, Golding SJ, Alvey C, Wass JA, Karpe F, Franks S \& McCarthy MI. Global adiposity rather than abnormal regional fat distribution characterizes women with polycystic ovary syndrome. Journal of Clinical Endocrinology and Metabolism 200893 999-1004. (doi:10.1210/jc.2007-2117)

Received 14 September 2015

Revised version received 2 December 2015

Accepted 15 December 2015 